\title{
Dilute acid saccarification of plant waste for producing green fuel
}

\author{
Ali Arasteh Nodeh*, Rasool Ghasemzadeh
}

\begin{abstract}
In this study, the dilute acid saccarification of plant waste as a lignocellulose biomass for fermentable sugar production as a green fuel has investigated. The Influence of 3 major parameters Temperature, acid concentration and time on produced fermentable sugars has been investigated by response surface model. According to previous studies levels for pretreatment were: temperature $\left(124,132\right.$ and $\left.140^{\circ} \mathrm{C}\right)$, process time (30,40 and 50 minute and sulfuric acid concentration $(1,2,3 \%)$. For this purpose, plant waste was gathered in November of 2015 from Torbat Heidarieh city (Razavi Khorasan, Iran). It was washed by distillated water, air dried and milled to particle size smaller than 50 micrometers and stored in sealed plastic bags at the room temperature. This prepared biomass heated in acid solution in autoclave for determined time. For determination of product filtered solution was analyzed by HPLC and results (Glucose) were modeled by a quadratic equation. This model shows Glucose concentration was influenced by quadratic power of acid concentration and time.
\end{abstract}

Keywords - Fermentable sugar, Saccharification, Plant, Hydrolysis

\section{Introduction}

As Henry Ford had stated, "The fuel of the future is going to come from apples, weeds, sawdust - almost anything. There is fuel in every bit of vegetable matter that can be fermented." Current industrial processes for bioethanol production use sugarcane (southern Hemisphere) or cereal grain (Northern Hemisphere) as feedstock, but they have to compete directly with the food sector [1]. Although these are the predominant feedstock that are used today, projected fuel demands indicate that new alternative low priced feedstock are needed to reduce ethanol production costs[2].since the price of feedstock contributes more than $55 \%$ to the production cost, inexpensive feedstock such as lignocelluloses. biomass and agri-food wastes, are being considered to make bioethanol competitive in open market[3]. Lignocelluloses biomass comprises divers groups of material: agricultural residues (cereal straw, rice hulls, cotton wastes, etc.), forestry residues (pruning and sawmill) and municipal solid wastes. Bioethanol production from these feed stocks could be an attractive alternative for disposal of these residues [4]. Ethanol derive from lignocelluloses is produced via four major consecutive steps: pretreatment, hydrolysis, fermentation, and separation [5].

These steps consist of depolymerization of holocellulose polymer into monomeric fermentable substrate, fermentation of depolymerized substrates, and the distillation of the fermentation broth to obtain dehydrated ethanol.

Department of chemical engineering, Quchan Branch, Islamic Azad University, Quchan, Iran
Basically, the lingocellulosic biomass comprises of cellulose, hemicelluloses and lignin [6]. Cellulose is a linear, crystalline homo polymer with a repeating unit of glucose strung together beta-glucosidic linkages. The structure is rigid and harsh treatment is required to break it down [7]. Hemi-cellulose consists of short, linear and highly branched chains of sugars. In contrast to cellulose, which is a polymer of only glucose, a hemicellulose is a hetero-polymer of Dxylose, D-glucose, D-galactose, D-mannose and L-arabinose [8]. The composition of holocellulose (cellulose + hemicellulose) varies with the origin of the lignocellulosic material. Ethanol production has been taken into estimation depending upon the ratio of hexosans (glucan, galactan and mannan) and pentosans (xylan, arabinan) in each bio mass source[9]. Xylose is believed to present in the largest amount in hemicelluloses. Unlike cellulose, hemicelluloses have a random and amorphous structure, which makes it easily be hydrolyzed by acid or base. Lignin is third major component in wood and comprises the glue that protects woody biomass from foreign invasion. The amount of the carbohydrate polymers and lignin depend on type of material. The hard woods such as white birch, aspen, red maple and oak contain $39-45 \%$ cellulose, $14-37 \%$ hemicelluloses, and $17-30 \%$ lignin. The corresponding values for softwoods e.g. pines and firs, are 41-50\% cellulose, 11-27 \% hemicelluloses and 20-3-\% lignin[10].

The carbohydrate polymers in the lignocellosic material need to be converted to simple sugars before fermentation, through a process called hydrolysis. However, several products can result from hydrolysis. There are several possible methods to hydrolyze lignocelluloses. The most commonly applied method can be classified in two groups: chemical hydrolysis and enzymatic hydrolysis. Cellulose and hemicelluloses can be converted to ethanol, while lignin remains as a by-product.

There are two types of acid hydrolysis process commonly used - dilute and concentrated acid hydrolysis. The dilute acid process is conducted under high temperature and pressure and has reaction time in the range of seconds or minutes. The concentrated acid process uses relatively mild temperatures, but at high concentration of sulfuric acid and a minimum pressure involved, which only creates by pumping the materials from vessel to vessel. Reaction times are typically much longer than for dilute acid process [9]. Sulfuric acid is the most investigated acid [11], although other acids such as $\mathrm{HCl}$ [12] have also been used.

Hydrolysis of lignocelluloses by concentrated sulfuric or hydrochloric acids is a relatively old process. Braconnot in 1819 first discovered that cellulose can be converted to fermentable sugar by concentrated acids [13]. Concentratedacid processes are generally reported to give higher sugar yield (e.g. $90 \%$ of theoretical glucose yield) and consequently higher ethanol yield, compared to dilute-acid processes. Furthermore, the concentrated-acid processes can operate at low temperature (e.g. $40^{\circ} \mathrm{C}$ ), which is a clear advantage compared to dilute-acid processes. However, the 
concentration of acid is very high in this method (e.g. 30$70 \%$ ), and dilution and heating of the concentrated acid during the hydrolysis process make it extremely corrosive. Therefore, the process requires either expensive alloys or specialized non-metallic constructions, such as ceramic or carbon-brick lining. The acid recovery is an energydemanding process. In addition, when sulfuric acid is used, the neutralization process produces large amounts of gypsum.

Cellulose, hemicellulose and lignin are broken down to mainly glucose, mannose or xylose, and phenolic compounds during acid hydrolysis, respectively. As soon as the monomers are produced, further decompositions occur during these process conditions yielding other unexpected compounds such as 5-hydroxymethyl furfural (HMF) from hexoses, and furfural from pentose. HMF and furfural are also decomposed into mainly levulinic acid and formic acid. A large number of aliphatic acids are present in acid hydrolyzates originated from wood extractives, lignin degradation and sugar degradation [14]. Acetic acid is a major acid constituent in hydrolyzates and is mainly produced from degradation of the acetyl group in the polysaccharides, whereas levulinic acid and formic acid are products of sugar degradation. For the fermentation process, the presence of acetic acid and furfural in hydrolyzates can hinder or prevent a subsequent fermentation step. Therefore, hydrolysates with low concentrations of inhibitors are required.

For fuel ethanol production, production fermentable sugars have been studies as a key step for effective utilization of lignocellosic biomass feedstock. This reaction is very complex. The substrate is in a solid phase and the catalyst in a liquid phase. The mechanism of the hydrolysis reaction includes $[15,16,17]$ : (i) diffusion of protons through the wet lignocellulosic matrix; (ii) protonation of the oxygen of a heterocyclic ether bond between the sugar monomers; (iii) breaking of the ether bond; (iv) generation of a carbocation as intermediate; (v) solvation of the carbocation with water; (vi) regeneration of the proton with cogeneration of the sugar monomer, oligomer or polymer depending on the position of the ether bond; (vii) diffusion of the reaction products in the liquid phase if it is permit for their form and size; (viii) restarting of the second step. Therefore, due to the difficult in modelling these processes, empirical models have been developed[18].

\section{Material and Methods}

Dilute acid hydrolysis was used to hydrolysis the plant waste as a lignocellulose biomass for fermentable sugar production. The influence of temperature, acid concentration, and reaction time on glucose production was studied. Results were formulated by using quadratic equation:

$\mathrm{Y}=\mathrm{A}_{0}+\mathrm{A}_{1} \cdot \mathrm{C}+\mathrm{A}_{2} \cdot \mathrm{T}+\mathrm{A}_{3} \cdot \mathrm{t}+\mathrm{A}_{4} \cdot \mathrm{C}^{2}+\mathrm{A}_{5} \cdot \mathrm{T}^{2}+\mathrm{A}_{6} \cdot \mathrm{t}^{2}+\mathrm{A}_{7} \cdot \mathrm{C} \cdot \mathrm{T}++$ $A_{8}$.C.t+ A. T.t
This equation allows influence of each factor on responses as well as interactions among factors to be determined. This equation has 9 constant coefficients and at least 9 equations was needed to determine this coefficients by least square methods. Based on previous experience with concentrated acid hydrolysis to ensure a broad range of response, Three levels for each factor were considered and Plant was pretreated at 9 different operational conditions according to Taguchi matrix. Selected conditions were shown in Table 1. Every experiment was run for three times and nearest results was averaged[19].

For hydrolysis using dilute acid hydrolysis, dried Plant was treated with 1 to $3 \mathrm{wt} \%$ sulfuric acid in screwcapped laboratory bottles (Pyrex bottles) at $65-95{ }^{\circ} \mathrm{C}$ for 30-50 hours with agitation by a laboratory mixer. After reaction time, sodium hydroxide $(\mathrm{NaOH})$ was used until its $\mathrm{PH}$ become around 7.0 and then solids were separated by filtering, washed with distilled water and final solution reached to $1000 \mathrm{ml}$. A $200 \mathrm{ml}$ sample of solution was used to analyze by HPLC.

TABLE I. EXPERIMENTAL DESIGN, EXPERIMENTAL FACTOR AND CODE LEVELS

\begin{tabular}{|c|c|c|c|c|}
\hline Run & $\begin{array}{c}\text { Acid } \\
\text { concentrat. } \\
(\boldsymbol{\%})\end{array}$ & $\begin{array}{c}\text { Time } \\
(\mathbf{m i n})\end{array}$ & $\begin{array}{c}\text { Tempe } \\
\text { rature } \\
\left({ }^{\mathbf{}} \mathbf{C}\right)\end{array}$ & $\begin{array}{c}\text { Result } \\
(\mathbf{g r} / \mathbf{l i t}) \\
(\text { glucose) }\end{array}$ \\
\hline 1 & 1 & 30 & 124 & 2.47 \\
\hline 2 & 2 & 40 & 124 & 5.31 \\
\hline 3 & 3 & 50 & 124 & 4.25 \\
\hline 4 & 2 & 30 & 132 & 8.01 \\
\hline 5 & 3 & 40 & 132 & 8.85 \\
\hline 6 & 1 & 50 & 132 & 7.11 \\
\hline 7 & 3 & 30 & 140 & 13.88 \\
\hline 8 & 1 & 40 & 140 & 7.19 \\
\hline 9 & 2 & 50 & 140 & 13.52 \\
\hline
\end{tabular}

The composition of the hydrolysate from acid hydrolysis (Glucose, Xylose) was determined by high performance liquid chromatography (HPLC). An HPLC model JASCO are used. Glucose analysis by Biorad column Aminex HPX$87 \mathrm{P}$ and detect by RI detector in temperature 40C and Acetic acid.

The knowledge of contribution of individual factors is a key to deciding the nature of the control to be established on a production process. The analysis of variance (ANOVA) is the statistical treatment most commonly applied to the results of the experiment to determine the percent contribution of each factor. Study of the ANOVA table for a given analysis helps to determine which of the factors need control and which do not. 


\section{Results and Discussion}

The study of pretreatment performance by concentrated acid was addressed by performing the experimental design in which process temperature $\left(\mathrm{T},{ }^{\circ} \mathrm{C}\right)$, process time $(\mathrm{t}, \mathrm{min})$, and acid concentration $(\mathrm{C}, \%)$, as detailed before, were retained as factors and glucose concentration $(\mathrm{G})$ was considered as response $(\mathrm{Y})$. The static interpretation of results was formulated by equation 1, which allows the influence of each factor on the response as well as interactions among factors to be determined, according to constant coefficients $\mathrm{A}_{\mathrm{i}}$. Table 2 summarizes the model constant coefficients obtained from ANOVA table for different responses. Fig. 1 shows a good agreement between predicted and experimental values.

TABLE II.

COEFFICIENT OF MATHEMATICAL MODEL EQ.(1)

\begin{tabular}{|c|c|}
\hline $\mathrm{A}_{0}$ & 184.53 \\
\hline $\mathrm{A}_{1}$ & 0 \\
\hline $\mathrm{A}_{2}$ & -1.8919 \\
\hline $\mathrm{A}_{3}$ & -2.5281 \\
\hline $\mathrm{A}_{4}$ & -1.1445 \\
\hline $\mathrm{A}_{5}$ & 0.006995 \\
\hline $\mathrm{A}_{6}$ & 0.008332 \\
\hline $\mathrm{A}_{7}$ & -0.1509 \\
\hline $\mathrm{A}_{8}$ & 0.097189 \\
\hline $\mathrm{A}_{9}$ & 0.012741 \\
\hline
\end{tabular}

Glucose extraction has a parabola curve versus Acid Concentration (fig. 2). That means Glucose production increase when acid concentration increased.

The effect of temperature on glucose production is shown in figure 1 . Increasing the hydrolysis temperature significantly affected Glucose production. The concentration of these materials increased with the increase in hydrolysis temperature. Temperature is second parameter in glucose production (fig 5).

Glucose production increases by reaction time (Fig 2).

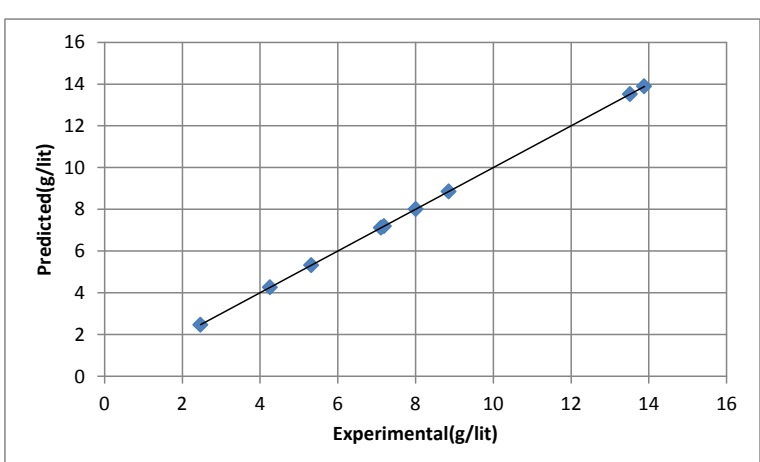

Figure 3 . Predicted versus experimental values for glucose of liquid phase after pretreatment

\section{Conclusions}

Regarding the experimental results, it can be concluded that this material can be used as a lignocelluloses material for bio ethanol production. This result can generalize to most of lignocelluloses that have the same structure and composition. In best condition, up to $14 \mathrm{gr} / \mathrm{lit}$ fermentable sugar could extraction from Plant wood. This fermentable sugar can ferment to bioethanol by yeast, if the concentrations of inhibitors (furfural) are not critical for the microorganism that will be grown on the hydrolysate. The optimum condition for hydrolysis of Plant wood peaked at acid concentration $3 \%$, Temperature $40^{\circ} \mathrm{C}$, Residence time $50 \mathrm{~min}$. Fermentable sugar in this condition is predicted about $14 \mathrm{gr} / \mathrm{lit}$ by models. The developed models are useful tools for further technical and economic studies.

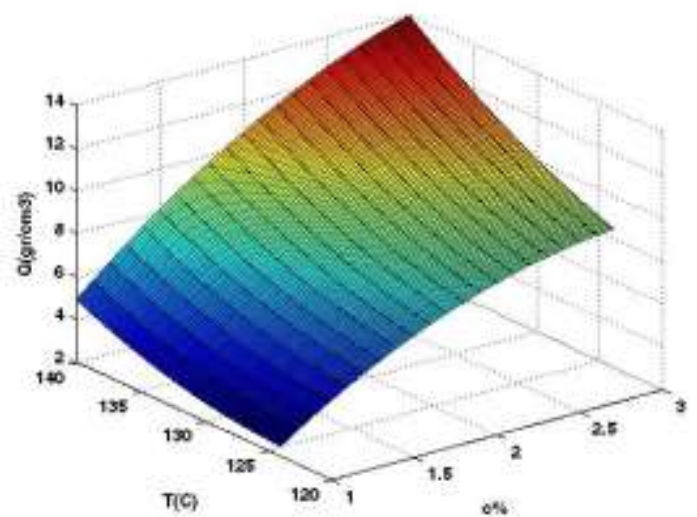

Figure 1. . Response surface for glucose recovery in liquid phase as a function of acid concentration and temperature according to the model

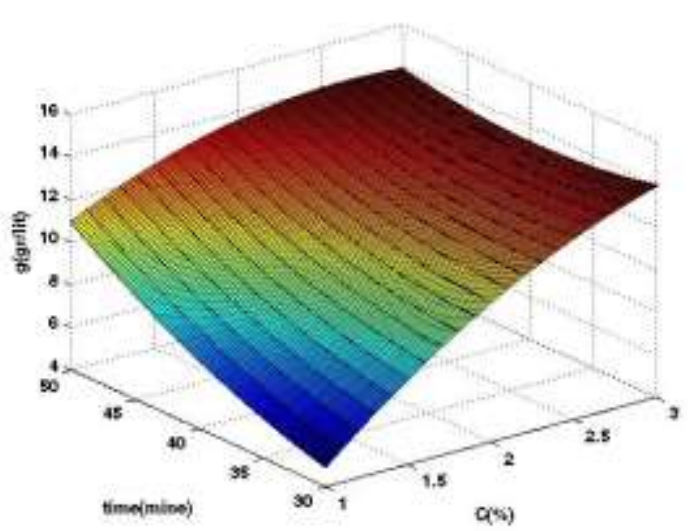

Figure 2. Response surface for glucose recovery in liquid phase as a function of acid concentration and time according to the model

\section{References}

[1] Wheals, A.E., Basso, t.c, Alves, D.M.G., Amorim, H.V., Fuel ethanol after 25 years, Trends Biotechnol. 17 (1999) 482-487.

[2] Palmarola-Adrados, B., Choteborska, P., Galbe, M., Zacchi, G., Ethanol production from non-starch carbohydrates of wheat bran. Biores. Technol. 96 (2005) 843-850

[3] Lichts,F.O.,Ethanol Production Costs: A Worldwide Survey. Special Report No. 132.2004

[4] Wyman, Ch.E., Twenty years of trials, tribulations, and research progress in bioethanol technology, Appl. Biochem. Biotech. 91(2001) $5-21$.

[5] Prabuddha Bansal et al., Modeling cellulose Kinetics on lignocellulosic substrates, 27 (2009) 833-848

[6] Hayn M, Klinger $\mathrm{R}$, Esterbauer $\mathrm{H}$,. Isolation and partial characterization of a low molecular weight endoglucanase from Trichoderma reesei. Suominen, P. and Reinikainen, T Proceedings of the second TRICEL symposium on Trichoderma reesei cellulases and 
Proc. of the International Conference on Advances in Bio-Informatics and Environmental Engineering - ICABEE 2016.

Copyright (C) Institute of Research Engineers and Doctors. All rights reserved.

ISBN: 978-1-63248-100-9 doi: 10.15224/ 978-1-63248-100-9-15

other hydrolases. Helsinki, Foundation for Biotechnical and Industrial Fermentation Research. (1993) 147-151.

[7] Gray KA, Zhao Land Emptage. Bioethanol. Curr Opin Chem Bioi, 10 (2006) 141-146.

[8] Shama. Developments in bioreactors for fuel ethanol production. Proc. Biochem. 23 (1988) 138-145.

[9] Anuj Kumar Chandel et al. Economics and environmental impact of bioethanol production technologies, Biotechnology and Molecular Biology Review 2 (2007) 14-32

[10] Taherzadeand Karimi "Bioethanol review,"BioResources 2 (2007) 472-499.

[11] Harris,E.,Beglinger, E., Hajny, G., and Sherrard, E., Hydrolysis of Wood:Treatment with sulfuric acid in a stationary digerster, Ind. Eng. Chem. 3 (1945) 12-23

[12] Hashem, A.M. and Rashad, M.M, Production of ethanol by yeast grown on hydrolyate of Egyptian sweet potato,Wgept Journal of Food Scince 21(1993) 171-180

[13] Sherrard, E., and Kressman, F, Review of processes in the United States prior to World War II, Ind.Eng.Chem. 37 (1945) 5-8.

[14] A Orozco, M. Ahmad, D. Rooney, G.M. Walker, Dilute acid hydrolysis of cellulose and cellulosic bio-waste using a microwave reactor system, Process Safety and Environmental Protection Trans IChemE, Part B 85 (2007) 446-449.

[15] carasco, F,et al,Fundamentos de la production de furfural, Afinidal, 48 (1991) 183-189

[16] Fengel, D.,et al, wood: chemistry, ultrastructure,reaction. Berlin:walter de Gruyter. 1984

[17] Harris,e.E.,Wood hydrolysis in Wood chemistry", Van Nostrand Renhold, New York, 1952,852

[18] R. Aguilar et al, Kinetic study of acid hydrolysis of sugar cane bagasse, Journal of food engineering, 55 (2002) 309-318

[19] Ranjit R.Roy, A primer on the Taguchi method, van nostrand einhold, first edition, 1990.

About Author (s):

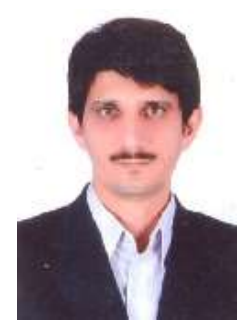

Ali Arasteh Nodeh

Chemical Engineering- Biotechnology

Islamic Azad University

Quchan Branch

Quchan-Iran 